\title{
The Laplacian spread of Cactuses
}

\author{
Ying Liul \\ College of Mathematics and Information, Shanghai Lixin University of Commerce, Shanghai, 201620, China \\ E-mail liuying@lixin.edu.cn
}

received 4 April 2009, revised 10 March 2010, 19 March 2010, 20 March 2010, accepted 20 Mar 2010.

Connected graphs in which any two of its cycles have at most one common vertex are called cactuses. In this paper, we continue the work on Laplacian spread of graphs, and determine the graph with maximal Laplacian spread in all cactuses with $n$ vertices.

Keywords: Cactus, Laplacian spread, Laplacian eigenvalues

\section{Introduction}

Let $G=(V, E)$ be a simple connected graph with vertex set $V=\left\{v_{1}, v_{2}, \cdots, v_{n}\right\}$ and edge set $E$. Denote by $d_{i}$ (or $d\left(v_{i}\right)$ ) the degree of the vertex $v_{i}$ of the graph $G$. Let $A(G)$ be the adjacency matrix of $G$ and $L(G)=D(G)-A(G)$ be the Laplacian matrix of the graph $G$, where $D(G)=\operatorname{diag}\left(d_{1}, d_{2}, \cdots, d_{n}\right)$ denotes the diagonal matrix of vertex degrees of $G$. It is easy to see that $L(G)$ is a positive semidefinite symmetric matrix and its rows sum to 0 , so $L(G)$ is singular. Denote its eigenvalues by $\mu_{1}(G) \geq \mu_{2}(G) \geq$ $\cdots \geq \mu_{n}(G)=0$, which are always enumerated in non-increasing order and repeated according to their multiplicities. We call the largest eigenvalue of $L(G)$ the Laplacian spectral radius of the graph $G$, denoted by $\mu(G)$. Moreover, since $G$ is connected if and only if $\mu_{n-1}(G)>0$, Fielder [2, 3] thought, in a sense, $\mu_{n-1}(G)$ as a quantitative measure of connectivity. Hence he called $\mu_{n-1}(G)$ the algebraic connectivity of $G$ and denoted by $\alpha(G)$. The Laplacian spread of the graph $G$ is defined to be

$$
\varphi_{L}(G)=\mu(G)-\alpha(G) .
$$

The notion of Laplacian spread is very significant in characterizing the global structural property of graphs. Recently, Fan et al [4] have showed that, among all trees with $n$ vertices, $K_{1, n-1}$ and $P_{n}$ are the unique trees with the maximal Laplacian spread and minimal Laplacian spread, respectively. Bao [1] et al determined the graph with maximal in all unicyclic graphs.

Connected graphs in which any two of its cycles have at most one common vertex are called cactuses. Throughout this paper, the characteristic polynomial $\operatorname{det}(x I-L(G))$ is denoted by $\Phi(G ; x)$, or simply $\Phi(G)$. If $v \in V(G)$, let $L_{v}(G)$ be the principal submatrix of $L(G)$ obtained by deleting the

\footnotetext{
${ }^{\dagger}$ Research supported by the National Natural Science Foundation of China 10871166. 


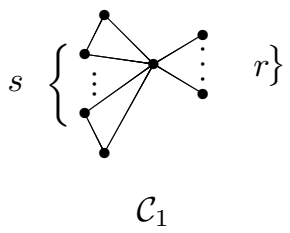

Fig. 1:

row and column corresponding to the vertex $v$. Let $\mathcal{C}_{n}$ be the set of cactuses with $n$ vertices. In this paper, we continue the work on Laplacian spread of graphs, and determine the graph class $\mathcal{C}_{1}$ (see Fig. 1) with maximal Laplacian spread in all $\mathcal{C}_{n}(n \geq 10)$, where $\mathcal{C}_{1}$ is the graph class by gluing one vertex of $s$ triangles to the maximum degree vertex of $K_{1, r}(2 s+r=n-1)$, respectively.

Now, we give the main theorem in this paper:

Theorem 1 Let $G \in \mathcal{C}_{n}-\left\{\mathcal{C}_{1}\right\}(n \geq 5)$, then $\quad \varphi_{L}(G)=\mu(G)-\alpha(G)<n-1=\varphi_{L}\left(\mathcal{C}_{1}\right)$.

\section{Proof of Theorem 1}

The following lemmas are necessary for our main results. Denote by $N(v)$ the neighborhood of the vertex $v \in V(G)$.

Lemma 2 ([[10]) Let $G$ be a connected graph, then

$$
\mu(G) \leq \max \left\{d\left(v_{i}\right)+d\left(v_{j}\right)-\left|N\left(v_{i}\right) \cap N\left(v_{j}\right)\right|: v_{i} \neq v_{j}\right\} .
$$

Lemma 3 ([5],9]) Let $G$ be a connected graph on $n$ vertices with at least one edge, then $\mu(G) \geq \Delta(G)+$ 1 , where $\Delta(G)$ is the maximum degree of the graph $G$, with equality if and only if $\Delta(G)=n-1$.

Lemma 4 ([8]) Suppose $u, v$ are two distinct vertices of a connected graph $H$. Let $G$ be the graph obtained from $H$ by attaching s new paths $u u_{i 1} u_{i 2} \cdots u_{i p_{i}}(i=1, \cdots, s)$ at $u$ and $t$ new paths $v v_{j 1} v_{j 2} \cdots v_{j p_{j}}$ $(j=1, \cdots, t)$ at $v$, respectively. Suppose $\Delta(G) \geq 3$. Let $X$ be a unit eigenvector of $G$ corresponding to $\mu(G)$. Let

$$
R_{G}(u, W, v)=G-v v_{11}-v v_{21}-\cdots-v v_{t 1}+u v_{11}+u v_{21}+\cdots+u v_{t 1}
$$

where

$$
W=\left\{v_{11}, \cdots, v_{t 1}\right\}
$$

Then

$$
\mu(G) \leq \max \left\{\mu\left(R_{G}(u, W, v)\right), \mu\left(R_{G}\left(v, W^{\prime}, u\right)\right)\right\}
$$

where $W^{\prime}=\left\{u_{11}, \cdots, u_{s 1}\right\}$ and this inequality is strict if $\left|x_{u}\right| \neq\left|x_{v}\right|$.

Lemma 5 ([7]) Let $G$ be a connected graph of $n$ vertices and with a cutpoint $v$. Then

$$
0<\alpha(G) \leq 1,
$$

the equality holds if and only if $v$ is adjacent to every other vertex of $G$. 
Lemma 6 [11] Let $G$ be a connected graph on $n$ vertices. Suppose that $v_{1}, v_{2}, \cdots, v_{s}(s \geq 2)$ are pairwisely non-adjacent vertices of $G$ and $N\left(v_{1}\right)=N\left(v_{2}\right)=\cdots=N\left(v_{s}\right)$. Let $G_{t}$ be a graph obtained from $G$ by adding any $t\left(0 \leq t \leq \frac{s(s-1)}{2}\right)$ edges among $v_{1}, v_{2}, \cdots, v_{s}$, then we have $\mu(G)=\mu\left(G_{t}\right)$.

Lemma 7 ([6]) Let $G=G_{1} u: v G_{2}$ be the graph obtained by joining the vertex $u$ of the graph $G_{1}$ to the vertex $v$ of the graph $G_{2}$ by an edge. Then

$$
\Phi(L(G))=\Phi\left(L\left(G_{1}\right)\right) \Phi\left(L\left(G_{2}\right)\right)-\Phi\left(L\left(G_{1}\right)\right) \Phi\left(L_{v}\left(G_{2}\right)\right)-\Phi\left(L\left(G_{2}\right)\right) \Phi\left(L_{u}\left(G_{1}\right)\right) .
$$

Lemma 8 Let $G \in \mathcal{C}_{n}$ and $u, v \in V(G)$, then

(1) If $u, v$ are adjacent, then $d(u)+d(v) \leq n+1$;

(2) If $u, v$ are not adjacent, then $d(u)+d(v) \leq n$.

Proof: Since for any $u, v \in V(G)$, we have $\quad d(u)+d(v)-|N(u) \cap N(v)|=|N(u) \cup N(v)| \leq n$, then

(1) If $u, v$ are adjacent, we know $\quad|N(u) \cup N(v)| \leq n,|N(u) \cap N(v)| \leq 1$, so $d(u)+d(v) \leq n+1$.

(2) If $u, v$ are not adjacent, we know $\quad|N(u) \cup N(v)| \leq n-2,|N(u) \cap N(v)| \leq 2$, so $d(u)+d(v) \leq n$.

From 1 , Lemma 3 and by Lemma 5 , for $G \in \mathcal{C}_{1}$ we obtain $\mu(G)=n$ and $\alpha(G)=1$, respectively. Then $\varphi_{L}(G)=\mu(G)-\alpha(G)=n-1$, so

$$
\varphi_{L}\left(\mathcal{C}_{1}\right)=\mu\left(\mathcal{C}_{1}\right)-\alpha\left(\mathcal{C}_{1}\right)=n-1 .
$$

Combining (1) and Lemma 2, we know if $G \in \mathcal{C}_{n}-\left\{\mathcal{C}_{1}\right\}$ and $|N(u) \cup N(v)| \leq n-1$ for all $u, v \in V(G)$, then $\mu(G) \leq n-1$, thus

$$
\varphi_{L}(G)=\mu(G)-\alpha(G)<n-1=\varphi_{L}\left(\mathcal{C}_{1}\right) .
$$

So in the followings we may always assume that there exist $u, v \in V(G)$ such that

$$
d(u)+d(v)-|N(u) \cap N(v)|=|N(u) \cup N(v)|=n .
$$

We introduce some notations. Let $T_{i}(p, q)(i=1,2,3)$ be a tree obtained from $P_{i+1}$ by adding $p, q$ pendent edges to a pendent vertex, respectively.

Each unicyclic graph can be obtained by attaching rooted trees to the vertices of a cycle $C_{k}$ of length $k$. Thus if $R_{1}, \cdots, R_{k}$ are $k$ rooted trees (of orders $n_{1}, \cdots, n_{k}$, say), then we adopt the notation $U_{k}\left(R_{1}, \cdots, R_{k}\right)$ (or simply $U\left(R_{1}, \cdots, R_{k}\right)$ sometime for convenience) to denote the unicyclic graph $G$ (of order $n=n_{1}+\cdots+n_{k}$ ) obtained by attaching the rooted tree $R_{i}$ to the vertex $v_{i}$ of a cycle $C_{k}=v_{1} v_{2} \cdots v_{k} v_{1}$ (i.e., by identifying the root of $R_{i}$ with the vertex $v_{i}$ ) for $i=1, \cdots, k$.

In order to prove our main theorem, we give three graph classes $\mathcal{G}_{1}, \mathcal{G}_{2}$ and $\mathcal{G}_{3}$. 


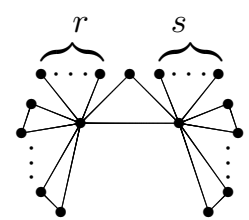

$\mathcal{G}_{1}$

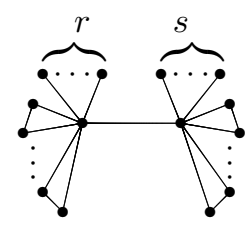

$\mathcal{G}_{2}$

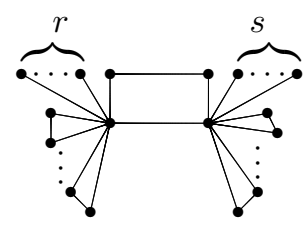

$\mathcal{G}_{3}$

Fig. 2:

Lemma 9 Let $G \in \mathcal{C}_{n}(n \geq 5)$ and $G \in\left\{\mathcal{G}_{1}, \mathcal{G}_{2}, \mathcal{G}_{3}\right\}$, then

$$
\varphi_{L}(G)=\mu(G)-\alpha(G)<n-1=\varphi_{L}\left(\mathcal{C}_{1}\right) .
$$

Proof: If $G \in \mathcal{G}_{1}$, by Lemma 6 and Lemma 4 , we have $\mu(G) \leq \mu\left(U_{3}(n-4,1,0)\right.$. Using Lemma 7 it is not difficult to calculate $\Phi(G)=x(x-1)^{n-5} f(x)$, where $f(x)=\left[x^{4}-(n+5) x^{3}+(6 n+3) x^{2}-\right.$ $(9 n-5) x+3 n]$. And if $n \geq 5$,

$$
\begin{gathered}
f\left(\frac{3}{7}\right)=\frac{3}{2401}(133 n+1868)>0, f(1)=-n+4<0, f(2)=n-2>0, \\
f(n-1)=-n+4<0, f\left(n-\frac{4}{7}\right)=\frac{1}{2401}\left(1029 n^{3}-7938 n^{2}+14924 n-2012\right)>0,
\end{gathered}
$$

then we have

$$
n-1<\mu(G)<n-\frac{4}{7}, \frac{3}{7}<\alpha(G)<1 .
$$

So $\varphi_{L}(G)=\mu(G)-\alpha(G)<n-1=\varphi_{L}\left(\mathcal{C}_{1}\right)$.

Using the similar method, we can obtain the same result.

If $G \in \mathcal{G}_{2}$, by Lemma 6 and Lemma 4 , we have $\mu(G) \leq \mu\left(T_{1}(n-3,1)\right)$. Using Lemma 7 , it is not difficult to calculate $\Phi(G)=x(x-2)(x-1)^{n-4} h(x)$, where $h(x)=\left[x^{3}-(n+2) x^{2}+(3 n-2) x-n\right]$. And if $n \geq 5$,

$$
\begin{gathered}
h\left(\frac{2}{7}\right)=-\frac{1}{343}(77 n+244)<0, h(1)=n-3>0, \\
h(n-1)=-1<0, h\left(n-\frac{5}{7}\right)=\frac{1}{343}\left(98 n^{2}-434 n+15\right)>0,
\end{gathered}
$$

then we have

$$
n-1<\mu(G)<n-\frac{5}{7}, \frac{2}{7}<\alpha(G)<1 .
$$

So $\varphi_{L}(G)=\mu(G)-\alpha(G)<n-1=\varphi_{L}\left(\mathcal{C}_{1}\right)$.

If $G \in \mathcal{G}_{3}$, by Lemma 6 and Lemma 4 , we have $\mu(G) \leq \mu\left(U_{4}(n-4,0,0,0)\right)$. Using Lemma7 7 it is not difficult to calculate $\Phi(G)=x(x-2)(x-1)^{n-5} g(x)$, where $g(x)=\left[x^{3}-(n+3) x^{2}+(4 n-2) x-2 n\right]$. And if $n \geq 5$,

$$
g\left(\frac{4}{7}\right)=-\frac{2}{343}(7 n+322)<0, g(1)=n-4>0, g(4)=-2(n-4)<0,
$$




$$
g(n-1)=-2<0, g\left(n-\frac{3}{7}\right)=\frac{2}{343}\left(98 n^{2}-476 n+39\right)>0,
$$

then we have

$$
n-1<\mu(G)<n-\frac{3}{7}, \frac{4}{7}<\alpha(G)<\frac{5}{7} .
$$

So $\varphi_{L}(G)=\mu(G)-\alpha(G)<n-1=\varphi_{L}\left(\mathcal{C}_{1}\right)$.

Lemma 10 Let $G \in \mathcal{C}_{n}-\left\{\mathcal{C}_{1}\right\}(n \geq 5), u, v \in V(G)$ and there exist $u, v \in V(G)$ such that

$$
d(u)+d(v)-|N(u) \cap N(v)|=|N(u) \cup N(v)|=n .
$$

Then

$$
\varphi_{L}(G)=\mu(G)-\alpha(G)<n-1=\varphi_{L}\left(\mathcal{C}_{1}\right)
$$

Proof: We discuss two cases according to $u v \in E(G)$ or $u v \notin E(G)$. If $u v \notin E(G)$, from the proof of Lemma 8 follows that $|N(u) \cup N(v)| \leq n-2$, which contradicts the assumptions of $|N(u) \cup N(v)|=n$, so we only discuss the case $u v \in E(G)$.

If $u v \in E(G)$, then by Lemma 8 , we have $|N(u) \cap N(v)| \leq 1$.

C1se 1: $|N(u) \cap N(v)|=1$.

Then there exist $r, s(r \geq s \geq 1)$ such that $G \in \mathcal{G}_{1}$ (see Fig. 2). By Lemma 9 , we have if $n \geq 5$, then $\mu(G)<n-1$.

C2se 2: $|N(u) \cap N(v)|=0$.

Then $G \in\left\{\mathcal{G}_{2}, \mathcal{G}_{3}\right\}$ (see Fig. 2). If $G \in \mathcal{G}_{2}$ for some $r, s$, by Lemma 9 we have for $n \geq 5$, $\mu(G)<n-1$.

If $G \in \mathcal{G}_{3}$ for some $r$, s, by Lemma 9 , we have for $n \geq 5, \mu(G)<n-1$.

From the above lemmas, we give the proof of Theorem 1

Proof of Theorem 1; From Lemma 5, we know $0<\alpha(G) \leq 1$.

If there exists $u, v \in V(G)$ such that $|N(u) \cup N(v)|=n$, then by Lemma 10 we have

$$
\varphi_{L}(G)=\mu(G)-\alpha(G)<n-1=\varphi_{L}\left(\mathcal{C}_{1}\right) .
$$

If $|N(u) \cup N(v)| \leq n-1$ for all $u, v \in V(G)$, then $\mu(G) \leq n-1$ following that Lemma 2 , so

$$
\varphi_{L}(G)=\mu(G)-\alpha(G)<n-1=\varphi_{L}\left(\mathcal{C}_{1}\right) .
$$

The proof is completed. 


\section{References}

[1] Y. H. Bao, Y. Y. Tan and Y. Z. Fan, The Laplacian spread of unicyclic graphs, Applied Mathematics Letters (2009), doi: 10.1016/j.aml.2009.01.023.

[2] M. Fielder, Algebra connectivity of graphs, Czechoslovake Math. J, 23(98)(1973) 298-305.

[3] M. Fielder, A property of eigenvectors of nonnegative symmetric matrices and its application to graph theory, Czechoslovake Math. J, 25(98)(1975) 706-618.

[4] Y. Z. Fan, J. Xu, Y. Wang and D. Liang, The Laplacian spread of a tree, Discrete Mathmatics and Theoretical Computer Science, 10(1)(2008) 79-86.

[5] R. Grone and R. Merris, The Laplacian spectrum of graph II, SIAM J.Discrete Math., 7 (1994) 221229.

[6] Guo Ji-ming, On the second largest Laplacian eigenvalue of trees, Linear Algebra and its Application, 2005, 404: 251-261.

[7] S. Kirkland, A bound on algebraic connectivity of a graph in trems of the number of cutpoints, Linear Multilinear Algebra, 47 (2000) 93-103.

[8] Y. Liu, J. Y. Shao and X. Y. Yuan, Some results on the Laplacian spectral radius of unicyclic graphs, Discrete Applied Math., 156 (2008) 2679-2697.

[9] R. Merris, Laplacian matrices of graphs: a survey, Linear Algebra Appl., 197-198 (1994) 143-176.

[10] O. Rojo, R. Soto and H. Rojo, An always nontrivial upper bound for Laplacian graph eigenvalues, Linear Algebra Appl., 312 (2000) 155-159.

[11] J. Y. Shao, J. M. Guo, H. Y. Shan, The ordering of trees and connected graphs by algebraic connectivity, Linear Algebra. Appl., 428 (2008) 1421-1438. 